Pacific Journal of Mathematics

SEMIPERFECT RINGS WITH ABELIAN GROUP OF UNITS 


\section{SEMIPERFECT RINGS WITH ABELIAN GROUP OF UNITS}

\section{W. K. NICHOLSON}

In 1963, Gilmer characterized all finite commutative rings with a cyclic group of units and, in 1967, Eldridge and Fischer generalized these results to rings with minimum condition. In the present paper these results are extended to semiperfect rings and generalizations of the three theorems are obtained. It is shown that a semiperfect ring with cyclic group of units is finite and is either commutative or is the direct sum of a commutative ring and the $2 \times 2$ upper triangular matrix ring over the field of two elements. Let $R$ be semiperfect with an abelian group of units. It is shown that $R$ is finite if either the group of units is finite or the group of units is finitely generated and the Jacobson radical is nil.

The proofs of all these results depend on our main theorem: The structure of a semiperfect ring $R$ with an abelian group of units is described completely up to the structure of commutative local rings. (That is commutative rings with a unique maximal ideal.) The groups of units of these local rings are shown to be direct factors of the group of units of $R$.

1. Preliminaries. Throughout this paper we assume that all rings are associative and have an identity and that all modules are unital. If $R$ is a ring we denote its group of units by $R^{*}$ and its Jacobson radical by $J(R)$. The ring of residues of the integers modulo $n$ will be denoted by $\mathscr{\varkappa}_{n}$. The following notions will be referred to several times below.

Definition 1. Let $R_{1}, R_{2}, \cdots, R_{n}$ be rings and, if $i \neq j$, let $X_{i j}$ be an $R_{i}-R_{j}$ bimodule. We define the semidirect sum $\left[R_{i}, X_{i j}\right]$ to be the ring of all $n \times n$ "matrices" $\left(x_{i j}\right)$ where $x_{i i} \in R_{i}$ for each $i$ and $x_{i j} \in X_{i j}$ for all $i \neq j$. These are added componentwise and we define the product $\left(x_{i j}\right)\left(y_{i j}\right)=\left(z_{i j}\right)$ as follows:

$$
\begin{array}{ll}
z_{i i}=x_{i i} y_{i i} & \text { for all } i=1,2, \cdots, n, \\
z_{i j}=x_{i i} y_{i j}+x_{i j} y_{j j} & \text { for all } i \neq j .
\end{array}
$$

It is easy to verify that $\left[R_{i}, X_{i j}\right]$ is an associative ring. If the bimodules $X_{i j}$ are all zero the semidirect sum $\left[R_{i}, X_{i j}\right]$ reduces to the usual direct sum $R_{1} \oplus R_{2} \oplus \cdots \oplus R_{n}$. More generally, we have that $\left[0, X_{i j}\right]$ is an ideal of $\left[R_{i}, X_{i j}\right]$ which squares to zero, and the quotient ring is isomorphic to $R_{1} \oplus R_{2} \oplus \cdots \oplus R_{n}$. Clearly the direct sum of two of these semidirect sums is again a semidirect sum. 
Definition 2. Let $R$ be a ring. A left $R$-module $X$ is said to be $G$-unital if $u x=x$ for all $u \in R^{*}$ and all $x \in X$. A bimodule is called $G$-unital if it is $G$-unital as a left and as a right module.

Clearly 0 is a G-unital module and submodules and quotient modules of such modules are again of the same type. If $X$ is a $G$ unital $R$-module we have $x+x=0$ for every $x \in X$ since $-1 \in R^{*}$. In other words $X$ is an elementary abelian 2-group.

Proposition 1. Let $R_{1}, R_{2}, \cdots, R_{n}$ be rings and let $X_{i j}$ be an $R_{i}-R_{j}$ bimodule for all $i \neq j$. The semidirect sum $\left[R_{i}, X_{i j}\right]$ has the following properties:

(1) $\left[R_{i}, X_{i j}\right]^{*}=\left\{\left(x_{i j}\right) \mid x_{i i} \in R_{i}^{*}\right.$ for each $\left.i\right\}$.

(2) $\left[R_{i}, X_{i j}\right]^{*}$ is abelian if and only if each $R_{i}^{*}$ is abelian and each $X_{i j}$ is G-unital.

(3) If $\left[R_{i}, X_{i j}\right]^{*}$ is abelian it is isomorphic to the direct product of all the multiplicative groups $R_{i}^{*}$ and all the additive groups $X_{i j}$.

Proof. (1) If $\left(x_{i j}\right)$ is given and $x_{i i} \in R_{i}{ }^{*}$ for each $i$ it is easy to verify that $\left(x_{i j}\right)^{-1}=\left(y_{i j}\right)$ where $y_{i i}=x_{i i}^{-1}$ for each $i$ and $y_{i j}=-x_{i i}^{-1} x_{i j} x_{j j}^{-1}$ for all $i \neq j$. The converse is clear.

(2) Suppose $\left[R_{i}, X_{i j}\right]^{*}$ is abelian. Then (1) and the definition of multiplication in $\left[R_{i}, X_{i j}\right]$ imply that each $R_{i}^{*}$ is abelian. Now choose arbitrary elements $x_{i i}, y_{i i} \in R_{i}^{*}$ for each $i$ and $x_{i j}, y_{i j} \in X_{i j}$ for all $i \neq j$. The units $\left(x_{i j}\right)$ and $\left(y_{i j}\right)$ commute so, for all $i \neq j$ :

$$
x_{i i} y_{i j}+x_{i j} y_{j j}=y_{i i} x_{i j}+y_{i j} x_{j j} \text {. }
$$

If $x_{i j}=0$ and $x_{j j}=1$ this shows $x_{i i} y_{i j}=y_{i j}$. Similarly $x_{i j} y_{j j}=x_{i j}$ and it follows that each $X_{i j}$ is $G$-unital.

Conversely: If $\left(x_{i j}\right),\left(y_{i j}\right) \in\left[R_{i}, X_{i j}\right]^{*}$ then, using (1), $x_{i i} y_{i i}=y_{i i} x_{i i}$. Furthermore, since the $X_{i j}$ are $G$-unital, we have

$$
x_{i i} y_{i j}+x_{i j} y_{j j}=y_{i j}+x_{i j}=x_{i j}+y_{i j}=y_{i i} x_{i j}+y_{i j} x_{j j}
$$

for all $i \neq j$ and it follows that $\left[R_{i}, X_{i j}\right]^{*}$ is abelian.

(3) This follows easily from the definition of multiplication in $\left[R_{i}, X_{i j}\right]$ and the fact that each $X_{i j}$ is $G$-unital.

Proposition 2. Let $R$ be a local ring. $(R / J(R)$ a divisor ring.)

(1) If the group of units of $R$ is abelian then $R$ is commutative.

(2) If $R$ possesses a nonzero $G$-unital module then $R / J(R) \cong \mathscr{\varkappa}_{2}$.

(3) If $R / J(R) \cong \mathscr{L}_{2}$ the G-unital $R$-modules are precisely the (additive) elementary abelian 2-groups.

Proof. (1) If $a, b \in J(R)$ then $1+a, 1+b \in R^{*}$ so $(1+a)(1+b)=$ 
$(1+b)(1+a)$. This implies $a b=b a$. If $a \in J(R)$ and $u \in R^{*}$ then $(1+a) u=u(1+a)$ so $a u=u a$.

(2) Let $X \neq 0$ be a $G$-unital left $R$-module. If $x \in X$ and $a \in J(R)$ then $(1+a) x=x$ so $a x=0$. Hence $X$ is a $G$-unital $R / J(R)$ module so we can assume $R$ is a division ring. But then if $0 \neq r \in R$ we have that $(1-r) x=0$ for every $x \in X$. Since $X \neq 0$ this means $1-r$ is not a unit so $r=1$. Hence $R \cong \mathscr{Z}_{2}$.

(3) If $X$ is an elementary abelian 2-group then it is a vector space over $\mathscr{Z}_{2}$. Since $R / J(R) \cong \mathscr{Z}_{2}, R$ acts on $X$ as follows: If $x \in X$ and $r \in R$ we have $r x=x$ if $r \in R^{*}$ and $r x=0$ if $r \in J(R)$. Clearly, $X$ is $G$-unital. Conversely: Every $G$-unital module is an elementary 2 -group since $-1 \in R^{*}$ and the action is as described.

In the next section we shall use these results to characterize the semiperfect rings $R$ where $R^{*}$ is abelian.

2. The main theorem. Throughout this section $R$ will denote a semiperfect ring with $R^{*}$ abelian. It is well known [2, Th. 20, p. 159] that $R$ is semiperfect if and only if we can write $1=e_{1}+$ $e_{2}+\cdots+e_{n}$ where the $e_{i}$ are orthogonal local idempotents. Hence each of the rings $e_{i} R e_{i}$ is local and, if $i \neq j$, the ring $e_{i} R e_{j}$ is an $e_{i} R e_{i}-e_{j} R e_{j}$ bimodule. For the moment let $\left[e_{i} R e_{j}\right]$ denote the set of all $n \times n$ "matrices" $\left(x_{i j}\right)$ with the $(i, j)$ entry $x_{i j}$ drawn from $e_{i} R e_{j}$. This is a ring if ordinary matrix operations are used.

Define a map $\phi: R \rightarrow\left[e_{i} R e_{j}\right]$ by $\phi(r)=\left(e_{i} r e_{j}\right)$ for each $r \in R$. Then $\phi$ is clearly a homomorphism of additive groups and it is a ring homomorphism since the $(i, j)$ entry of $\phi(r) \phi(s)$ is

$$
\sum_{k}\left(e_{i} r e_{k}\right)\left(e_{k} s e_{j}\right)=e_{i} r\left(e_{1}+e_{2}+\cdots+e_{n}\right) s e_{j}=e_{i} r s e_{j} .
$$

Moreover $\phi$ is one-to-one. Indeed, if $\phi(r)=0$ then $e_{i} r e_{j}=0$ for all $i, j$ and so $r=\sum_{i, j} e_{i} r e_{j}=0$. Finally $\phi$ is onto. For if $\left(e_{i} r_{i j} e_{j}\right) \in\left[e_{i} R e_{j}\right]$ is given let $r=\sum_{i, j} e_{i} r_{i j} e_{j}$. It is easy to check that $\phi(r)=\left(e_{i} r_{i j} e_{j}\right)$. Hence $\phi$ is a ring isomorphism and so we have represented $R$ as a generalized matrix ring. Our aim is to show that it is a semidirect sum.

Lemma 1. If $e_{i} \neq e_{j}$ and $e_{j} \neq e_{k}$ then $e_{i} R e_{j} R e_{k}=0$.

Proof. Let $x \in e_{i} R e_{j}$ and $y \in e_{j} R e_{k}$. Then $x^{2}=0$ (since $e_{i} e_{j}=0$ ) so $1+x$ is a unit. Similarly $1+y$ is a unit and so, since $R^{*}$ is abelian, $x y=y x$. But $x=e_{i} x$ and $y=e_{j} y$ so that $x y=e_{i} x y=e_{i} y x=$ $e_{i} e_{j} y x=0$.

It now follows easily that the multiplication in $\left[e_{i} R e_{j}\right]$ is that of 
the semidirect sum. Indeed if $\left(x_{i j}\right),\left(y_{i j}\right) \in\left[e_{i} R e_{j}\right]$ and we write $\left(x_{i j}\right)\left(y_{i j}\right)=\left(z_{i j}\right)$, then using the lemma:

$$
\begin{aligned}
& z_{i i}=\sum_{k} x_{i k} y_{k i}=x_{i i} y_{i i} \text { for all } i=1,2, \cdots, n, \\
& z_{i j}=\sum_{k} x_{i k} y_{k j}=x_{i i} y_{j i}+x_{i j} y_{j j} \text { for all } i \neq j .
\end{aligned}
$$

Hence, in the notation of $\S 1, R \cong\left[e_{i} R e_{i}, e_{i} R e_{j}\right]$. But then Proposition 1 shows that each $\left(e_{i} R e_{i}\right)^{*}$ is abelian and each $e_{i} R e_{j}$ is a $G$-unital bimodule. Since each $e_{i} R e_{i}$ is local, it is commutative by Proposition 2 . Furthermore, $e_{i}$ is central if and only if $e_{i} R e_{j}=0=e_{j} R e_{i}$ for all $j \neq i$. It follows that either $e_{i}$ is central or $e_{i} R e_{i}$ possesses a nonzero $G$-unital module. In the latter case $e_{i} R e_{i} / J\left(e_{i} R e_{i}\right) \cong \mathscr{Z}_{2}$ by Proposition 2 . This proves the "only if" part of the following theorem; the rest follows from Propositions 1 and 2.

THEOREM 1. Let $R$ be a semiperfect ring. The group of units of $R$ is abelian if and only if $R \cong T \oplus S$ where $T$ is zero or a direct sum of commutative local rings $R_{i}$ and $S$ is zero or $S \cong\left[L_{i}, X_{i j}\right]$. Here each $L_{i}$ is a commutative local ring with $L_{i} / J\left(L_{i}\right) \cong \mathscr{L}_{2}$ and each $X_{i j}$ is a G-unital $L_{i}-L_{j}$ bimodule. Moreover:

(1) The bimodules $X_{i j}$ can be chosen to be arbitrary elementary abelian 2-group where the action of $L_{i}$ is defined as follows: If $r \in L_{i}$ and $x \in X_{i j}$ set $r x=x$ if $r \in L_{i}^{*}$ and $r x=0$ if $r \in J\left(L_{i}\right)$.

(2) The group of units of $R$ is isomorphic to the direct product of all the groups $R_{i}^{*}$, all the groups $L_{i}^{*}$ and all the (additive) groups $X_{i j}$.

This characterizes $R$ completely up to the structure of the commutative local rings involved. The groups of units of these local rings inherit many properties from $R^{*}$ by $(2)$ and often this leads to a complete characterization. This will be exemplified in $\S 3$ below in the case where $R^{*}$ is assumed to be cyclic. Also, each of these local rings is a homomorphic image of $R$ so they inherit many ring-theoretic conditions which could be imposed on $R$, for example the descending chain condition.

An immediate consequence of Theorem 1 is that if $R$ is semiperfect and $R^{*}$ is abelian then $R / J(R)$ is a finite direct sum of fields. Of course this result follows from structure theory.

The next result is a generalization (in the case where $R$ has an identity) of a theorem of Eldridge and Fischer ([3], Th. 1, p. 244).

COROLlaRY 1. Let $R$ be a semiperfect ring with abelian group of units. Then $R$ is commutative if either of the following conditions is satisfied: 
(1) $2 x=0$ in $R$ implies $x=0$

(2) $R^{*}$ has no direct factor each element of which has order 2.

Proof. Let $\left[L_{i}, X_{i j}\right]$ be the semidirect sum appearing in the decomposition of $R$. If $x \in X_{i j}$ then $2 x=0$ since $X_{i j}$ is $G$-unital so each $X_{i j}=0$ if condition (1) holds. If condition (2) holds each $X_{i j}=0$ by (2) of the theorem. The result follows.

CoRollary 2. Let $R$ be a semiperfect ring. If the group of units of $R$ is abelian and finite then $R$ is finite.

Proof. By (2) of Theorem 1 each of the local rings appearing in the decomposition of $R$ has a finite group of units and each of the bimodules appearing in the semidirect sum is finite. But if $L$ is a local ring and $L^{*}$ is finite then $J(L)$ is finite since $1+J(L) \subseteq L^{*}$ and $L / J(L)$ is finite since $[L / J(L)]^{*} \cong L^{*} /(1+J(L))$. This implies $L$ is finite and the result follows.

A natural question is whether a semiperfect ring $R$ must be finite when $R^{*}$ is assumed to be abelian and finitely generated. The answer is yes if $R^{*}$ is cyclic (see Theorem 2 below) or if $J(R)$ is nil. The next result will be useful in both cases.

LEMma 2. Let $R$ be a commutative local ring. If $R^{*}$ is finitely generated then $R$ is noetherian, $R / J(R)$ is a finite field and $J(R)^{n} / J(R)^{n+1}$ is a finite ring for each $n$.

Proof. If $A_{1} \subseteq A_{2} \subseteq \cdots$ is a chain of (proper) ideals of $R$ we have the chain $1+A_{1} \subseteq 1+A_{2} \subseteq \cdots$ of subgroups of $R^{*}$. It follows that $R$ is noetherian. We have $[R / J(R)]^{*} \cong R^{*} / 1+J(R)$ so the field $R / J(R)$ has a finitely generated group of units. Hence it is finite. Finally, $J(R)^{n} / J(R)^{n+1}$ is a vector space over $R / J(R)$ and is finite dimensional since $R$ is noetherian.

We can now prove the following result which generalizes another result of Eldridge and Fischer ([3], Th. 2, p. 245).

Proposition 3. Let $R$ be a semiperfect ring with $R^{*}$ abelian. If $R^{*}$ is finitely generated and $J(R)$ is nil then $R$ is finite.

Proof. Decompose $R$ as in Theorem 1 and let $L$ be one of the commutative local rings which appear. Then $L^{*}$ is finitely generated by (2) of Theorem 1 and $J(L)$ is nil ( $L=e R e$ for some $e^{2}=e \in R$ ). Since $L$ is noetherian by Lemma 2 , write $J(L)=L a_{1}+L a_{2}+\cdots+L a_{n}$ where the $a_{i} \in J(L)$. Hence, if $m \geqq 1$ we have $J(L)^{m}=\sum L x_{1}^{k 1} a_{2}^{k 2} \cdots a_{n}^{k_{n}}$ 
where the sum is taken over all $k_{i} \geqq 0$ satisfying $k_{1}+k_{2}+\cdots+k_{n}=m$. Since $J(L)$ is nil this implies that $J(L)$ is nilpotent. But then Lemma 2 implies that $J(L)$, and hence $L$, is finite.

It remains to show that if $\left[L_{i}, X_{i j}\right]$ is the semidirect sum appearing in the decomposition of $R$ then each $X_{i j}$ is finite. But each $X_{i j}$ is finitely generated as an additive group and so, since it is a vector space over $\mathscr{Z}_{2}$, it is finite. This completes the proof.

We remark that the hypothesis that $J(R)$ is nil was used only to show that $J(L)$ is nilpotent.

3. Cyclic groups of units. Gilmer [4] has characterized all finite commutative rings with a cyclic group of units and Eldridge and Fischer [3] have extended these results to artinian rings. In order to cover the semiperfect case we need the following negative result.

Proposition 4. If $R$ is a commutative local ring the group of units of $R$ is not infinite cyclic.

Proof. Assume, on the contrary, that $R^{*}$ is infinite cyclic. Then the characteristic of $R$ is two since $(-1)^{2}=1$. By Lemma $2 R$ is noetherian and $R / J(R)$ is finite. Hence, $J(R)^{2} \neq J(R)$. But the additive group $J(R) / J(R)^{2}$ is cyclic since it is naturally isomorphic to the multiplicative group $1+J(R) / 1+J(R)^{2}$. Since the characteristic is two, it follows that $J(R) / J(R)^{2}$ has two elements. But $J(R) / J(R)^{2}$ is a vector space over the field $R / J(R)$ so $R / J(R) \cong \mathscr{z}_{2}$.

We now claim that $R$ is an integral domain. If not let $P$ be any prime ideal of $R$. Then $P \neq 0$ so $(R / P)^{*} \cong R^{*} / 1+P$ is finite cyclic. Since $R / P$ is local it follows that $R / P$ is a finite integral domain and hence that $P$ is maximal. Hence every prime ideal is maximal and so ([6], p. 203) $R$ is artinian. But then $R$ is finite by Proposition 3, a contradiction. Hence $R$ is an integral domain.

Now let $u$ be a generator of $R^{*}=1+J(R)$. Write $u=1+a$ and $u^{-1}=1+b$ where $a, b \in J(R)$. Then $1+a+a^{2}$ is a unit so $1+$ $a+a^{2}=(1+a)^{k}$ for some $k \in \mathscr{z}$. It is easy to check that $k=0,1,2$ are impossible. Suppose $k \geqq 3$. Then we have

$$
1+a+a^{2}=1+\gamma a+\delta a^{2}+a^{t} u
$$

where $3 \leqq t \leqq k, u$ is a unit and $\gamma$ and $\delta$ are each either 0 or 1 . Since $R$ is a domain it is easy to check that each of these possibilities for $\gamma$ and $\delta$ lead to a contradiction. Hence we must have $1+a+a^{2}=u^{-l}$ for some $l \geqq 1$. But $1+a+a^{2}=1+u+u^{2}$ and $1+b+b^{2}=1+$ $u^{-1}+u^{-2}$ as is easily verified. Hence 


$$
1+b+b^{2}=u^{-2}\left(1+a+a^{2}\right)=u^{-2} u^{-l}=(1+b)^{l+2} .
$$

This leads to a contradiction just as before and so completes the proof.

We can now obtain a generalization of another result of Eldridge and Fischer ([3], Th. 3, p. 248) and, in so doing, obtain a much easier proof of that result.

THEOREM 2. Let $R$ be a semiperfect ring with cyclic group of units. Then $R$ is finite and is either commutative or is isomorphic to the direct sum of a commutative ring and the ring of $2 \times 2$ upper triangular matrices over $\mathscr{Z}_{2}$.

Proof. Decompose $R$ as in Theorem 1. By (2) of Theorem 1, each of the local rings appearing has a cyclic group of units and, by Proposition 4, it is finite. It follows that each of these local rings is finite. Now let $\left[L_{i}, X_{i j}\right]$ be the semidirect sum appearing. Each $X_{i j}$ is cyclic as an additive group and so, since it is an elementary abelian 2-group, it has two elements. Hence $R$ is finite. Furthermore, the fact that all $X_{i j}$ are direct factors of $R^{*}$ means that at most one is nonzero. If all are zero then $\left[L_{i}, X_{i j}\right]$ is commutative so $R$ is commutative. If, without loss of generality, $X_{12} \neq 0$, we have

$$
\left[L_{i}, X_{i j}\right]=\left(\begin{array}{cc}
L_{1} X_{12} \\
0 & L_{2}
\end{array}\right) \oplus L_{3} \oplus \cdots \oplus L_{n}
$$

where $\left(\begin{array}{cc}L_{1} & X_{12} \\ 0 & L_{2}\end{array}\right)$ is a semidirect sum.

Moreover, each $L_{i}^{*}$ has odd order so $L_{i}$ has characteristic two. But then, if $a \in J\left(L_{i}\right)$, there exists an odd integer $n$ such that $1=(1+a)^{n}=1+a+a^{2} r$ where $r \in L_{i}$. Hence $a(1+a r)=0$ so $a=0$. This means $J\left(L_{i}\right)=0$ so $L_{i} \cong \mathscr{Z}_{2}$. In particular, $\left(\begin{array}{ll}L_{1} X_{12} \\ 0 & L_{2}\end{array}\right)$ is isomorphic to the ring of $2 \times 2$ upper triangular matrices over $\mathscr{F}_{2}$. This completes the proof.

This theorem completely characterizes the semiperfect rings with a cyclic group of units since the finite commutative local rings of this type have been characterized by Gilmer [4] and later by Ayoub [1] and Pearson and Schneider [5]. Gilmer cited the ring of $2 \times 2$ upper triangular matrices over $\mathscr{\varkappa}_{2}$ as an example of a finite noncommutative ring with cyclic group of units. Theorem 2 shows that this is essentially the only such semiperfect ring. 


\section{REFERENCES}

1. C. W. Ayoub, On finite primary rings and their groups of units, Compositio Math., 21 (1969), 247-252.

2. E. A. Behrens, Ring Theory, Academic Press, New York, 1972.

3. K. E. Eldridge and I. Fischer, D.C.C. rings with a cyclic group of units, Duke Math. J., 34 (1967), 243-248.

4. R. W. Gilmer, Finite rings with a cyclic multiplicative group of units, Amer. J. Math., 85 (1963), 447-452.

5. K. R. Pearson and J. E. Schneider, Rings with a cyclic group of units, J. of Algebra, 16 (1970), 243-251.

6. D. Zariski and P. Samuel, Commutative Algebra, Vol. I, Van Nostrand, Princeton, N. J., 1958.

Received August 4, 1972.

THE UNIVERSITY OF CALGARY 


\section{PACIFIC JOURNAL OF MATHEMATICS}

\section{EDITORS}

RICHARD ARENS (Managing Editor) University of California

Los Angeles, California 90024

R. A. Beaumon'T

University of Washington Seattle, Washington 98105
J. Dugundj1*

Department of Mathematics University of Southern California Los Angeles, California 90007

D. Gilbarg and J. Milgram Stanford University Stanford, California 94305

\section{ASSOCIATE EDITORS}

E. F. BECKENBACH

B. H. NeUMANN

F. WOLF

K. YOSHIDA

\section{SUPPORTING INSTITUTIONS}

UNIVERSITY OF BRITISH COLUMBIA CALIFORNIA INSTITUTE OF TECHNOLOGY UNIVERSITY OF CALIFORNIA MONTANA STATE UNIVERSITY UNIVERSITY OF NEVADA NEW MEXICO STATE UNIVERSITY OREGON STATE UNIVERSITY UNIVERSITY OF OREGON OSAKA UNIVERSITY

\section{UNIVERSITY OF SOUTHERN CALIFORNIA STANFORD UNIVERSITY UNIVERSITY OF TOKYO UNIVERSITY OF UTAH WASHINGTON STATE UNIVERSITY UNIVERSITY OF WASHINGTON AMERICAN MATHEMATICAL SOCIETY NAVAL WEAPONS CENTER}

* C. R. DePrima California Institute of Technology, Pasadena, CA 91109, will replace J. Dugundji until August 1974. 


\section{Pacific Journal of Mathematics}

\section{Vol. 49, No. $1 \quad$ May, 1973}

A. Bigard, Free lattice-ordered modules ...........................

Richard Bolstein and Warren R. Wogen, Subnormal operators in strictly cyclic

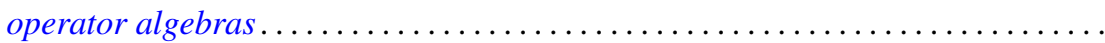

Herbert Busemann and Donald E. Glassco, II, Irreducible sums of simple

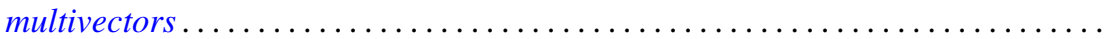

W. Wistar (William) Comfort and Victor Harold Saks, Countably compact groups

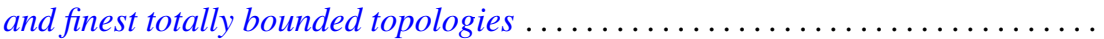

Mary Rodriguez Embry, Maximal invariant subspaces of strictly cyclic operator

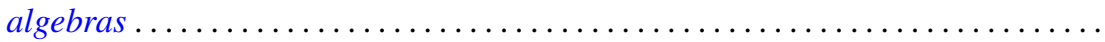

Ralph S. Freese and James Bryant Nation, Congruence lattices of semilattices......

Ervin Fried and George Grätzer, A nonassociative extension of the class of

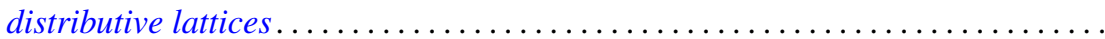

John R. Giles and Donald Otto Koehler, On numerical ranges of elements of locally

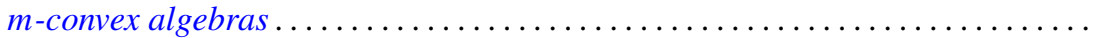

David A. Hill, On dominant and codominant dimension of $\mathrm{QF}-3$ rings ........ John Sollion Hsia and Robert Paul Johnson, Round and Pfister forms over $R(t) \ldots$ I. Martin (Irving) Isaacs, Equally partitioned groups . . . . . . . . . . . . . .

Athanassios G. Kartsatos and Edward Barry Saff, Hyperpolynomial approximation of solutions of nonlinear integro-differential equations.

Shin'ichi Kinoshita, On elementary ideals of $\theta$-curves in the 3-sphere and 2-links in the 4-sphere

Ronald Brian Kirk, Convergence of Baire measures

R. J. Knill, The Seifert and Van Kampen theorem via regular covering spaces ..

Amos A. Kovacs, Homomorphisms of matrix rings into matrix rings ..

Young K. Kwon, HD-minimal but no $H D$-minimal ..........

Makoto Maejima, On the renewal function when some of the mean renewal lifetimes are infinite

Juan José Martínez, Cohomological dimension of discrete modules over profinite groups.

W. K. Nicholson, Semiperfect rings with abelian group of units

Louis Jackson Ratliff, Jr., Three theorems on imbedded prime divisors of principal ideals.

Billy E. Rhoades and Albert Wilansky, Some commutants in $B(c)$ which are almost matrices

John Philip Riley Jr., Cross-sections of decompositions . . .

Keith Duncan Stroyan, A characterization of the Mackey uniformity $m\left(L^{\infty}, L^{1}\right)$ for

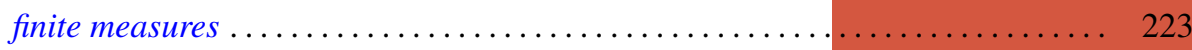

Edward G. Thurber, The Scholz-Brauer problem on addition chains . . . . . . . . . 229

Joze Vrabec, Submanifolds of acyclic 3-manifolds ............

Philip William Walker, Adjoint boundary value problems for compactified singular

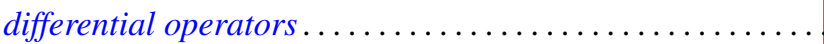

